\title{
DNA metabarcoding reveals the complex and hidden responses of chironomids to multiple stressors
}

\author{
Arne J. Beermann ${ }^{1,2^{*}}$, Vera M. A. Zizka ${ }^{1,2}$, Vasco Elbrecht ${ }^{1,2,3}$, Viktor Baranov ${ }^{4}$ and Florian Leese ${ }^{1,2}$
}

\begin{abstract}
Background: Chironomids, or non-biting midges, often dominate stream invertebrate communities in terms of biomass, abundance, and species richness and play an important role in riverine food webs. Despite these clear facts, the insect family Chironomidae is often treated as a single family in ecological studies or bioassessments given the difficulty to determine specimens further. We investigated stressor responses of single chironomid operational taxonomic units (OTUs) to three globally important stressors (increased salinity, fine sediment and reduced water flow velocity) in a highly replicated mesocosm experiment using a full-factorial design (eight treatment combinations with eight replicates each).

Results: In total, 183 chironomid OTUs (97\% similarity) were obtained by applying a quantitative DNA metabarcoding approach. Whereas on the typically applied family level, chironomids responded positively to added fine sediment and reduced water velocity in the streambed and negatively to reduced velocity in the leaf litter, an OTU-level analysis revealed a total of 15 different response patterns among the 35 most common OTUs only. The response patterns ranged from (a) insensitivity to any experimental manipulation over (b) highly specific sensitivities to only one stressor to (c) additive multiple-stressor effects and even (d) complex interactions.

Conclusion: Even though most OTUs (> 85\%) could not be assigned to a formally described species due to a lack of accurate reference data bases at present, the results indicate increased explanatory power with higher taxonomic resolution. Thus, our results highlight the potential of DNA-based approaches when studying environmental impacts, especially for this ecologically important taxon and in the context of multiple stressors.
\end{abstract}

Keywords: Taxonomic resolution, Chironomidae, OTU, Stressor responses, Field experiment

\section{Background}

Chironomidae ('non-biting midges') is a highly diverse family of insects with an estimated global richness of up to 20,000 species [11]. They occur in every zoogeographic region including Antarctica [4], and their larvae inhabit limnic, marine, terrestrial, and even subterranean environments $[1,13]$. Chironomids occur over a wide range of environmental gradients, including gradients of $\mathrm{pH}$, salinity, dissolved oxygen, water level, and temperature (reviewed in: $[3,56])$. In streams and lakes, they are

\footnotetext{
*Correspondence: arne.beermann@uni-due.de

${ }^{1}$ Aquatic Ecosystem Research, University of Duisburg-Essen, Universitätsstraße 5, 45141 Essen, Germany

Full list of author information is available at the end of the article
}

frequently the most abundant group of insects [56] often accounting for at least $50 \%$ of the total macroinvertebrate species [3]. Because of their species richness and high abundance, chironomids play an important role in aquatic and terrestrial food webs (e.g., [75]). Chironomidae larvae are found in all functional feeding groups (gatherers, filterers, scrapers, shredders, and predators) and many species are able to exhibit different feeding modes [6]. Chironomid larvae themselves are prey to fish [58] and many species of invertebrates (reviewed in [56]).

Despite their species richness, diversity, abundance, and ecological importance, the taxon Chironomidae has not experienced the same autecological in-depth research as other freshwater taxa [56]. Thus, despite 
detailed investigation of single species such as Chironomus riparius [3, 53] or Chironomus plumosus [30, 32], many ecological studies only treat Chironomidae at family or subfamily level. This divergence is partly attributed to their small body size and difficulties in the morphological identification of their larvae and adults, which hinders studying the autecology of species, especially in field studies. Beyond that, the taxonomic impediment combined with the high abundance of Chironomidae makes it often not feasible to use the taxon in ecological studies or for morphology-based routine biomonitoring below family or subfamily level. Even though for Chironomidae an increased taxonomic resolution can be achieved by studying pupal exuviae rather than larvae [24], exuviae drift downstream on the water and thus do not provide the exact same information on time and space as benthic sampling.

Identifying communities with many species using classical taxonomy demands increased time, money, and expertise (e.g., [33, 34]). Therefore, the concept of taxonomic sufficiency [21] was developed, which suggests using higher taxonomic levels than species as taxonomic surrogates. Consequently, many studies addressing the concept of taxonomic sufficiency revolve around the question if there is a loss of information when higher taxonomic levels are used (e.g., [37, 46, 68]). However, a precise alternative to morphological species identification is nowadays possible with molecular methods such as DNA barcoding [29] or its fast and cost-efficient extension DNA metabarcoding [66], which allows for simultaneous analysis of hundreds of specimens and species. Molecular methods are already widely used for species identification (e.g., $[8,28,73,76])$ and have been shown to be beneficial for bioassessments $[19,22,25,64]$. These new DNAbased techniques now also make it possible to test and even circumvent the concept of taxonomic sufficiency. They hold the potential to provide highly resolved data on community change under stressor conditions that is typically not assessed. The importance of this additional layer of resolution for ecosystem process understanding and subsequently management measures has been rarely explored so far. Key targets for such studies should be highly abundant, ecologically relevant and species-rich taxa like Chironomidae.

In this study, we selected Chironomidae as an ecological key taxon and investigated specific stressor responses of single Chironomidae operational taxonomic units (OTUs) - likely representing individual speciesobtained by DNA metabarcoding. The chironomids were obtained from a former mesocosm field experiment [5] that studied effects of three globally important stressors on stream communities. The tested stressors were increased salinity, added fine sediment, and reduced water flow velocity, alone and in all possible combinations. Among the macroinvertebrate taxa in the experiment, the Chironomidae were the most abundant group (59,325 specimens, $59.6 \%$ of all specimens), and due to their sheer abundance, they were identified morphologically only to family level. In the mesocosm experiment, two microhabitats were distinguished and analyzed separately: channel substratum (here referred to as 'streambed') and leaf litter. On family level, chironomid abundances of the streambed increased with added fine sediment and reduced water flow velocity. In the microhabitat leaf litter, chironomid abundance decreased upon flow velocity reduction, which combined with the streambed results could be interpreted as behavioral shift from the leaf litter to the streambed. Changes in salinity did not affect the Chironomidae abundances in any microhabitat [5].

In view of recent studies $[9,69]$, we expected to find a large number of different chironomid species/OTUs in the experiment. However, following the concept of taxonomic sufficiency, we predicted similar responses to the different stressors for the vast majority of chironomid species/OTUs as on family level (see above).

\section{Methods \\ Mesocosm experiment}

A multiple stressor mesocosm field experiment (original experiment design by [54]; see [5] for full experiment description) was conducted from 8 March to 22 April 2014 at the Felderbach (Germany, North Rhine-Westphalia, $51^{\circ} 20^{\prime} 59.09^{\prime \prime} \mathrm{N}, 7^{\circ} 10^{\prime} 14.03^{\prime \prime} \mathrm{E}, 136 \mathrm{~m}$ a.s.l). Stream water was continuously pumped into the experiment to maintain a constant water flow into each of 64 mesocosms $(25 \mathrm{~cm}$ diameter, volume $3.5 \mathrm{~L}$; Microwave Ring Moulds, Interworld, Auckland, New Zealand), which were arranged in four blocks of 16 mesocosms each. The mesocosms contained the two compartments channel substratum $(300 \mathrm{~mL}$ fine sediment $[<2 \mathrm{~mm}], 900 \mathrm{~g}$ gravel $[2-30 \mathrm{~mm}]$ and seven stones $[>30 \mathrm{~mm}])$ and leaf litter bags $(12.5 \times 6.5 \mathrm{~cm}, 5 \mathrm{~mm}$ mesh size, $2.5 \mathrm{~g}$ leafs $)$ of dried alder leafs, which resemble the two microhabitats streambed and leaf litter in the study. Colonization of mesocosms occurred via drift (water intake pump mesh size $4 \mathrm{~mm}$ ) and was complemented with macroinvertebrates from multi-habitat kick-net sampling (96 kick samples in total, 12 benthic kick samples per 8 channels, area of benthic habitat provided per individual channel: $0.163 \mathrm{~m}^{2}$ ). The experiment ran for 46 days (24-day colonization, 22-day manipulative period). Responses of macroinvertebrates to stressors were tested in a $2 \times 2 \times 2$ full-factorial design with two levels of each factor and 8 replicates per treatment combination (see [5] for details on the chosen factor levels): salinity (ambient [18.2 mg/L, $\mathrm{SD} \pm 4.1]$ 
versus increased [312.2 $\mathrm{mg} / \mathrm{L}, \mathrm{SD} \pm 78.5]$ chloride concentration), fine sediment (ambient $[300 \mathrm{~mL}<2 \mathrm{~mm}]$ versus added $[300 \mathrm{~mL}<2 \mathrm{~mm}$ plus $450 \mathrm{~mL}<0.5 \mathrm{~mm}]$ ), and water flow velocity (normal $[16.5 \mathrm{~cm} / \mathrm{s}, \mathrm{SD} \pm 0.1]$ versus reduced $[9.6 \mathrm{~cm} / \mathrm{s}, \mathrm{SD} \pm 0.1])$. Macroinvertebrates were sampled at the last day of the experiment by first taking out the leaf litter bags followed by sieving the channel substratum for macroinvertebrates. Macroinvertebrates from both microhabitats were stored separately for every single mesocosm in $96 \%$ ethanol (which was replaced with fresh $96 \%$ ethanol the same day) at $-20{ }^{\circ} \mathrm{C}$ until further processing. All macroinvertebrates were identified morphologically and counted. For the present study only Chironomidae, which were not identified further below family level, were used for further molecular analysis.

\section{DNA extraction, DNA metabarcoding, and bioinformatics}

For the molecular analysis, chironomids were obtained from both microhabitats (streambed, leaf litter) from all 64 mesocosms. The 128 samples were dried separately in $1.5 \mathrm{~mL}$ Eppendorf tubes on a heating block at $50{ }^{\circ} \mathrm{C}$ for $16-24 \mathrm{~h}$, before weighing their respective dry mass (Additional file 1). Specimens were grinded in their respective tube by five zirconia beads $(2.0 \mathrm{~mm}$ Zirconia Beads, BioSpec Products, Bartlesville, USA) at $6 \mathrm{~m} / \mathrm{s}$ for $3 \times 45 \mathrm{~s}$ on a FastPrep ${ }^{\circledR}-24$ tissue grinder (MP Biomedicals, Eschwege, Germany). DNA was extracted from the grinded tissue using a modified salt extraction protocol [65]; modified by [74], followed by an RNA digestion step using $1 \mu \mathrm{L}$ RNase A on $50 \mu \mathrm{L}$ sample for $30 \mathrm{~min}$ at $37{ }^{\circ} \mathrm{C}$ and a clean-up step using the NucleoSpin ${ }^{\circledR}$ Gel and PCR Clean-up kit (Macherey-Nagel, Düren, Germany) to minimize the concentration of possible inhibitors prior to PCR. DNA concentrations of the extracted and cleaned-up samples were measured using the Qubit 2.0 (Broad Range Kit, Thermo Fisher Scientific, Beverly, USA) and diluted to $25 \mathrm{ng} / \mu \mathrm{L}$. DNA was amplified using a two-step PCR protocol. In the first step, DNA was amplified using illustra puretaq ready-to-go PCR beads (GE Healthcare UK Limited, Little Chalfont, UK) with $12.5 \mathrm{ng}$ DNA and $0.5 \mu \mathrm{M}$ of each primer (BF2, BR2; [17]) filled up to $25 \mu \mathrm{L}$ with sterile $\mathrm{H}_{2} \mathrm{O}$ at: $94{ }^{\circ} \mathrm{C}$ for 3 min initial denaturation, followed by 25 cycles of $94{ }^{\circ} \mathrm{C}$ for $30 \mathrm{~s}$ denaturation, $50{ }^{\circ} \mathrm{C}$ for $30 \mathrm{~s}$ annealing, $72{ }^{\circ} \mathrm{C}$ for $2 \mathrm{~min}$ elongation, and final elongation at $72^{\circ} \mathrm{C}$ for $5 \mathrm{~min}$. PCR success was validated by gel electrophoresis before the PCR product was cleaned-up using the NucleoSpin ${ }^{\circledR}$ Gel and PCR Clean-up kit (Macherey-Nagel, Düren, Germany) to remove any bovine serum albumin (BSA) from the samples. In the second step, DNA was amplified using $1 \times$ Buffer, $0.2 \mathrm{mM}$ dNTPs, $0.5 \mu \mathrm{M}$ of each primer, $0.025 \mathrm{U} / \mu \mathrm{L}$ 5Prime HotMaster Taq DNA
Polymerase (Quantabio, Beverly, USA), $1 \mu \mathrm{L}$ DNA template from the first PCR step, filled up to $50 \mu \mathrm{L}$ with sterile $\mathrm{H}_{2} \mathrm{O}$. The above-described PCR protocol was applied, with 15 instead of 25 cycles, in the second step. Samples were individually tagged in the second PCR step using fusion primers ([17]; all primer combinations can be found in Additional file 2). DNA concentrations were measured after the second PCR step (Additional file 1) using the Fragment Analyzer (Standard Sensitivity NGS Fragment Analysis Kit; Advanced Analytical, Ankeny, USA). Two libraries were pooled including specimen abundance as a factor (Additional file 1). The first library consisted of all samples from the experimental blocks 1 and 4 and the second library of all samples from experimental blocks 2 and 3. Pooled this way, both libraries contained an equal amount of samples from both microhabitats $(32$ streambed and 32 leaf litter samples per library), an equal amount of samples per treatment (four replicates of each treatment combination) and nearly the same number of chironomid specimens $(29,915$ specimens [block 1 and 4] versus 29,410 specimens [block 2 and 3]). A left-sided size selection was performed for both libraries using $0.76 \times$ SPRI select (Beckman Coulter, Krefeld, Germany). Each library was sequenced using the MiSeq platform with a paired-end v2 kit (read length $2 \times 250 \mathrm{bp}$ ) at GATC, Germany (5\% PhiX spike into increase sequence diversity). Raw data were processed with R JAMP v023 (https://github.com/VascoElbrecht/JAMP). Sequences were assigned to their original sample using the module Demultiplexing_shifted, followed by paired-end merging using Usearch (v10.0.240, [14]) through the JAMP module U_merge_PE. Reverse complement sequences were generated where necessary and quality filtering was conducted (maxee $=1 ;$ [14]). Pre-processing of sequences included primer removal (Cutadapt v1.9; [43]) discarding of reads $\pm 10 \mathrm{bp}$ of the expected length and dereplication with removing singletons. Operational taxonomic unit (OTU) clustering was conducted ( $97 \%$ similarity) and clusters with at least $0.01 \%$ abundance in one sample (after mapping of the reads including singletons) were included for further analyses. After remapping the remaining reads of OTUs that were discarded in the first filtering step, a second filtering step was conducted for each sample separately, in which only OTUs with an abundance of at least $0.01 \%$ in the respective sample were retained. OTUs were assigned to a taxonomic group by comparison with the Barcode of Life Database (BOLD, http://www.boldsystems.org/; accessed 02.08.2017) as implemented in JAMP. Since a $4-5 \%$ threshold has been suggested to be appropriate to delineate species of the chironomid genus Tanytarsus [39], we carried out a second analysis using a 
combination of Usearch (v10.0.240; [14]) and Vsearch (v2.8.1; [60]) with a 5\% clustering threshold to obtain a more conservative estimation of the actual number of species.

\section{Conceptual model, read abundances, and statistics}

It is assumed that the initial chironomid communities in the experiment by Beermann et al. [5] were similar or at least did not vary systematically between the treatments. Differences in specimen abundances at the end of the experiment are considered to be the effect of the applied stressors and a shift of organisms from one microhabitat to the other to represent a behavioral shift. DNA metabarcoding fails at picking up the exact specimen abundances $[15,57]$, which are needed for arithmetic-mean-based statistics that are used for analyzing stressor effects. Species and specimens differ in their biomass, and, therefore, also in their amount of DNA [18]. These differences are further amplified in a PCR, a pattern which is also modified by differences in primer binding efficiency [15], ultimately distorting the use of read abundances as a proxy for specimen abundances. In this study, we used highly degenerated primers $[16,17]$ and focused on a single family. To test if the species, potentially being found in this study, can be recovered with the used primers, we clustered (97\% similarity, PrimerMiner v0.18; [16]) and aligned all publically available chironomid COI sequences from specimens sampled in Germany from BOLD (downloaded 01.06.2017, Additional file 3). We then tested the match of our used forward primer (BF2) sequence against all the available OTUs showing that the primer matched to $>98.5 \%$ of the OTUs without a single mismatch, supporting a generally good match. The reverse primer could not be tested as it is located in the HCO2198 binding region. Even though a primer bias between species cannot be excluded (i.e., certain species might be over- or underrepresented in their read abundance as a result of different primer binding efficiencies), this bias is expected to not systematically vary between the same OTUs in different treatments. A bias due to unequal biomass of specimens $[15,18]$ cannot be excluded, but is expected to be comparably small here, because chironomid specimens show much less variation in biomass among different species than other taxa such as Ephemeroptera, Plecoptera, and Trichoptera. Furthermore, when analyzing stressor effects, the number of reads of a single OTU is compared to the number of reads of the same OTU and not to those of other OTUs (with the exception of community variables, see below). Unless the different treatments of the experiment significantly impacted on growth rates during the experimental manipulation and thereby on organism size and biomass, the same OTUs under different treatments should contribute a comparable amount of DNA per specimen. We argue that read abundances can be used in our case as a proxy for real abundances. Even though our data do not allow for quantifying the mean number of individuals affected by a stressor treatment, we argue that the analysis of read abundances is sufficient to infer stressor responses of the respective OTUs. In addition, although a high variation in the number of reads per OTU and sample may be expected, this study is backed by a high number of replicates (8) per treatment combination, which makes the results robust against random variation. Hence, read abundances of OTUs were used as an input for statistical tests. Since the sequencing depth per sample can vary for technical reasons the number of reads was standardized (see Additional file 4) prior to statistical analyses. Data were analyzed using SPSS 23 (IBM SPSS Statistics; IBM Company, Chicago, IL, U.S.A.) and R v3.3.3 [67]. We examined the responses of three community metrics for both microhabitats: OTU richness, Simpson's index of diversity, and Pielou's evenness. For each metric, we performed an ANOVA with salinity, fine sediment and flow velocity as fixed factors. The ANOVA model was intercept (d.f. 1) + salinity (1) + fine sediment $(1)+$ velocity $(1)+$ salinity $\times$ fine sediment (1) + salinity $\times$ velocity $(1)+$ fine sediment $\times$ salinity $(1)+$ salinity $\times$ fine sediment $\times$ velocity $(1)+$ error $(56, n=64)$. Since null hypothesis significance testing does not provide any estimates of the magnitude of an effect of interest [47], we calculated standardized effect sizes for all results with $p \leq 0.10$ to allow evaluating the biological relevance of our findings (partial $\eta^{2}$ values, range $0-1$ ). To assess treatment effects on invertebrate community composition, we performed a MANOVA with the multivariate equivalent of the model above for all OTUs with a read abundance of at least 50,000 reads $(0.39 \%)$ for the total read abundance and at least 30,000 reads $(0.23 \%)$ for the respective microhabitat (35 OTUs in total, 24 OTUs for the streambed, and 25 OTUs for the leaf litter). Moreover, we examined the between-subjects effects in the MANOVA for each common taxon to determine their individual responses. After exploratory analysis, community-level and taxon data were log-transformed $(\log +1)$ to improve normality and homoscedasticity. OTU sequences of the analyzed 35 OTUs where compared to the Barcode of Life Database to explore the taxonomy of the OTUs found in this study. The criteria to accept a species name were at least $95 \%$ similarity to a reference sequence, $\geq 5$ published reference sequences, adult reference specimens 
and $\geq 1$ visible project with a visible specimen identifier (see Additional file 5 for details).

\section{Results}

A total of 20,598,800 reads were generated in both sequencing runs combined (Library 1: 10,277,200; Library 2: 10,321,600). After bioinformatic processing (13,012,030 reads remaining) and taxon assignment $12,975,968$ reads were identified as belonging to $183 \mathrm{Chi}$ ronomidae operational taxonomic units (OTUs, 97\% similarity; see Additional file 6 for reads belonging to other taxonomic groups). The alternative approach of using a $5 \%$ clustering threshold resulted in 142 OTUs, but will not be referred to from here on unless specifically mentioned. The average sequencing depth was 259 ( $\pm 67 \mathrm{SD})$ and $199( \pm 102 \mathrm{SD})$ reads/specimen for the streambed and leaf litter, respectively, before standardizing the reads for further analysis. The number of reads per sample correlated with the number of specimens per sample (Spearman's rho, $r_{\mathrm{s}}=0.88, p<0.001, n=128$ ). $31.3 \%$ of these reads are from streambed and $68.7 \%$ from leaf litter samples. A total of 35 OTUs (i.e., the most common OTUs) were analyzed for individual stressor responses (Tables 1 and 2), of which 14 OTUs were well represented (i.e., within the chosen read abundance threshold) in both microhabitats, while 10 OTUs were more exclusive (i.e., the chosen threshold was only met for one microhabitat) in the streambed and 11 OTUs in the leaf litter. Eight OTUs of the streambed (33\%) and 9 of the leaf litter $(36 \%)$ did not respond to the experimental manipulations. For the remaining OTUs, we observed 14 different response patterns (Tables 1 and 2) across both microhabitats. When comparing OTUs analyzed for both microhabitats, 3 OTUs (OTU 3, 7, 11) did not respond to any treatment combination in either microhabitat, 5 OTUs (OTUs 5, 16, 18, 262, and 466) responded to the experimental manipulation in one microhabitat but not the other and the remaining 6 OTUs (OTUs 1, 2, 4, 9, 17, and 21) showed different response patterns in the respective microhabitat. OTUs 1 (29.6\%), 2 (17.1\%), 3 (6\%), 4 (5.8\%), and 466 (5.1\%) were the most abundant OTUs across both microhabitats accounting for $>63 \%$ of the total reads.

\section{Streambed}

The community metrics OTU richness and Simpson's diversity increased upon fine sediment addition (Table 1, Fig. 1). The MANOVA revealed that streambed community composition (24 OTUs) was affected by added fine sediment and reduced flow velocity. Concordant with that result, fine sediment had an effect on 12 OTUs (50\%) and flow reduction on 9 OTUs (37.5\%), of which 5 OTUs responded to both factors in a double positive manner (additive effects; e.g., OTU 1, Table 1, Fig. 2). The effect of added fine sediment was positive for all affected OTUs except for OTU 16, which decreased in abundance upon fine sediment addition. All 9 OTUs affected by the manipulation in flow velocity increased in abundance when the flow velocity was reduced. It is notable that 7 out of 9 OTUs that responded to the change in flow velocity were OTUs being more exclusive to the streambed than the leaf litter. Only one OTU (OTU 4, Fig. 2) responded to salinity manipulation by increasing with higher salinity. Positive synergistic interactions between salinity and sediment (OTU 6), salinity and velocity (OTU 6, OTU 25) and fine sediment and velocity (OTU 4) were found, i.e., the respective OTUs showed a higher increase in abundance than expected based on the single stressor effects. A complex three-way interaction affected OTU evenness. While reduced flow and fine sediment addition affected OTU evenness negatively at ambient salinity and positively at increased salinity, all three stressors combined had a positive antagonistic effect (i.e., less positive than predicted additively; following the terminology by [55]).

\section{Leaf litter}

The OTU richness decreased upon flow reduction in the leaf litter (Table 2, Fig. 1). Manipulation of flow velocity had an effect on 13 OTUs (52\%), which all decreased upon flow reduction, and concordantly, the MANOVA revealed that flow velocity had an effect on the community composition (25 OTUs). The abundance of two OTUs (OTU 4 and 9) increased with higher salinity, while the abundance of one OTU (OTU 5) decreased. Fine sediment affected one OTU (OTU 30) resulting in a higher abundance upon fine sediment addition. A single two-way interaction was observed for OTU 9. OTU 9 decreased in abundance upon flow reduction and fine sediment addition, but responded in a negative antagonistic (i.e., less negative than predicted additively; [55]) way when both stressors where combined. Two complex three-way interactions were observed for OTU 21 and OTU 17. At ambient salinity, OTU 21 decreased in abundance with reduced flow and added fine sediment, but increased when both stressors where combined. At increased salinity, OTU 21 still decreased upon flow reduction, but increased with added fine sediment and most notably decreased when all three stressors where combined. OTU 17 decreased with reduced flow and added fine sediment, but increased when both stressors where combined at ambient salinity. These effects were reversed at increased salinity, i.e., OTU 17 increased upon flow reduction and fine sediment addition and decreased when all three stressors were present. 


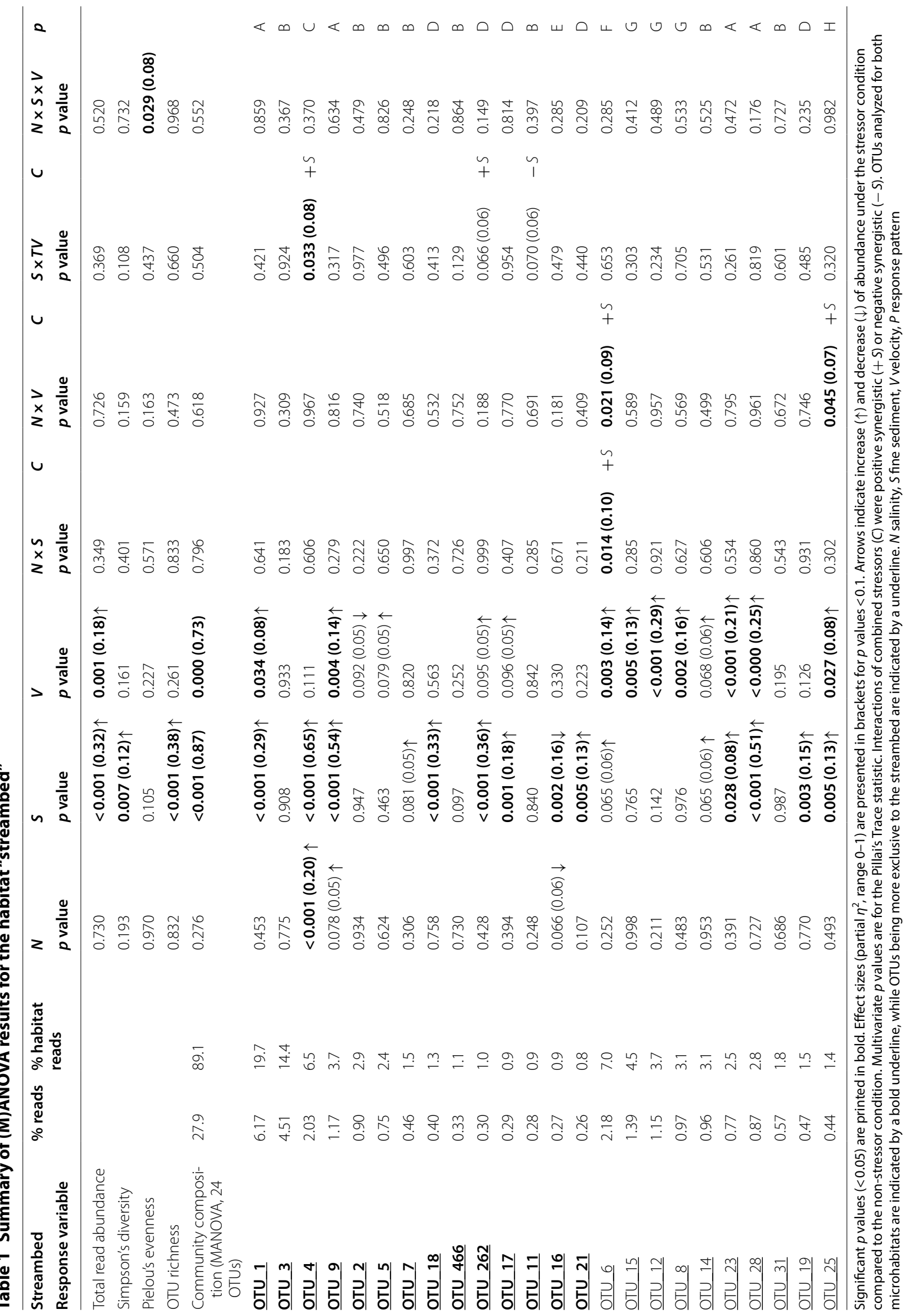




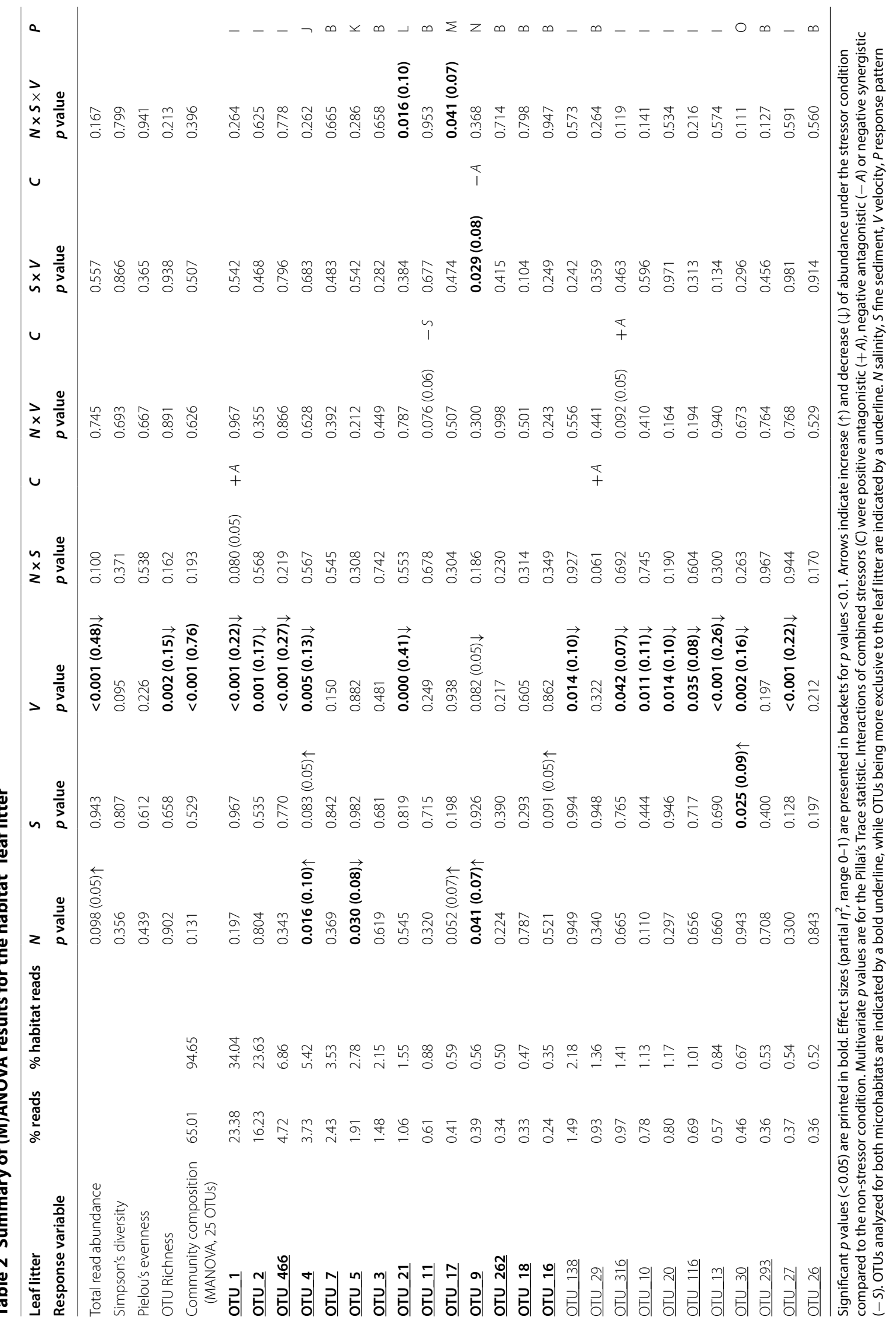



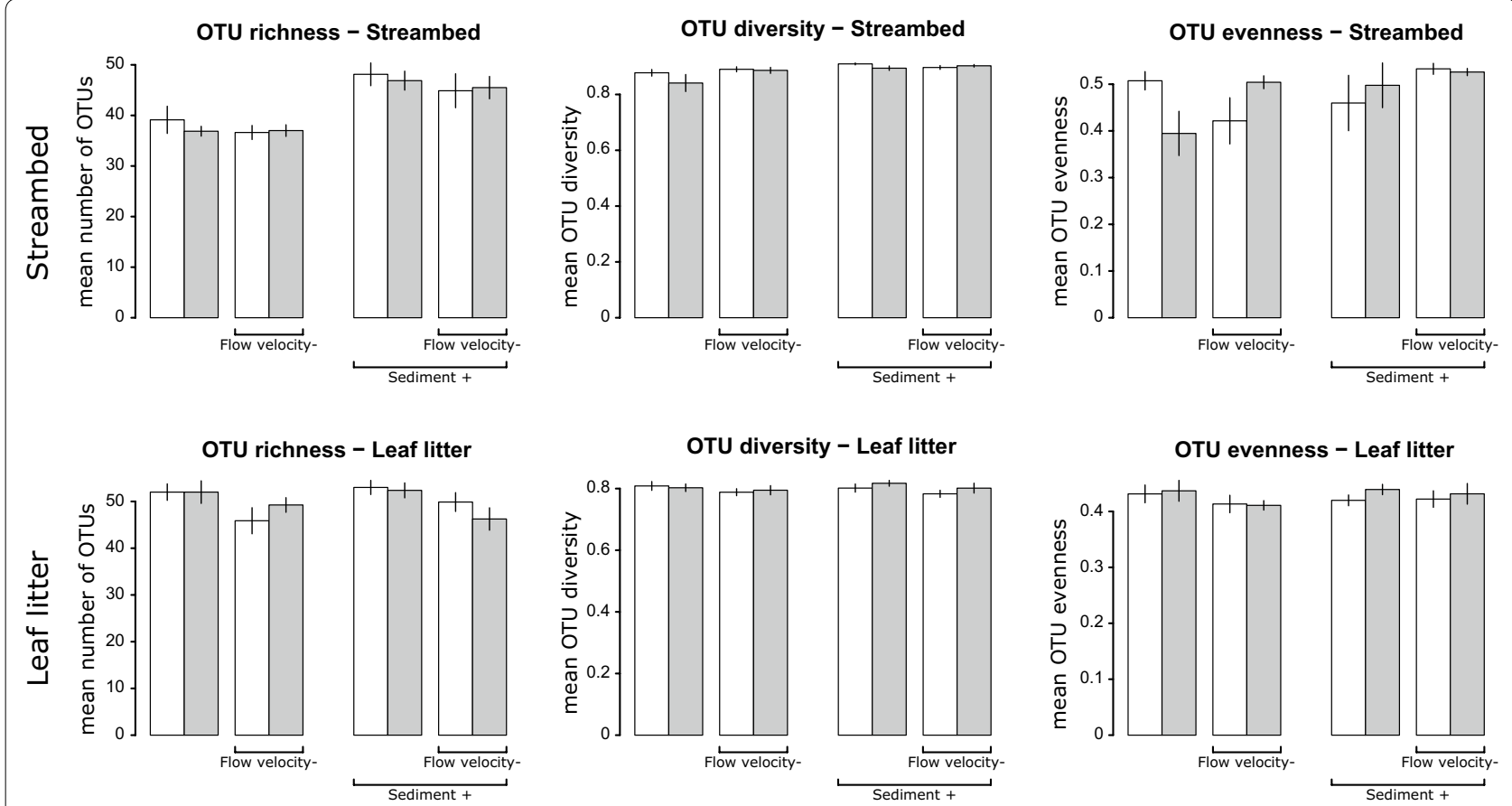

Fig. 1 Community metrics OTU richness, diversity, and evenness of chironomid OTUs in both microhabitats: streambed and leaf litter. White bars represent ambient and grey bars increased salinity. Error bars represent standard errors. Sample size for each treatment combination is $n=8$
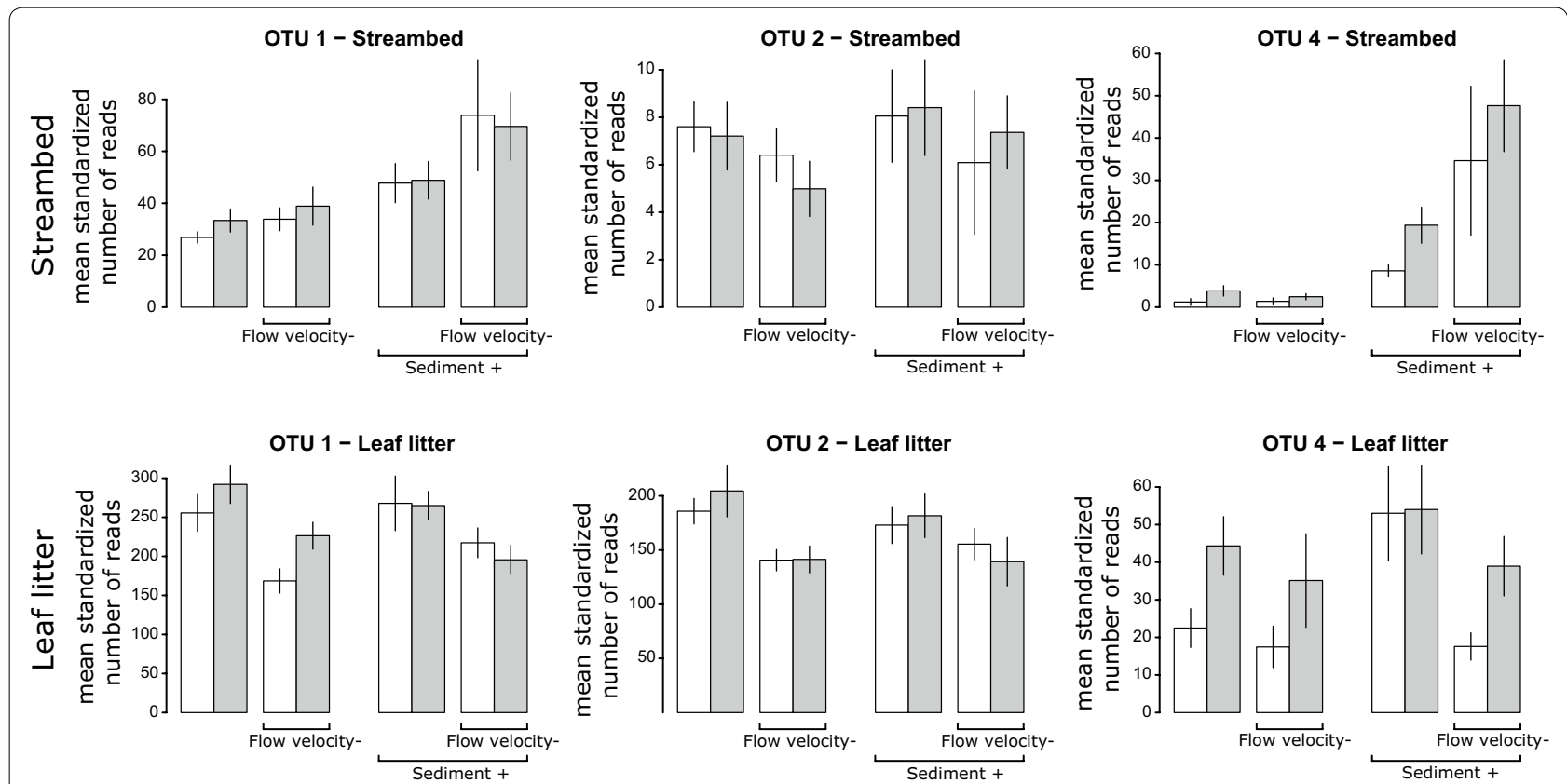

Fig. 2 Read abundances of OTU 1, OTU 2, and OTU 4 in their respective microhabitats: streambed and leaf litter. White bars represent ambient and grey bars increased salinity. Error bars represent standard errors. Sample size for each treatment combination is $n=8$

\section{Taxon name assignment}

Based on the available reference sequences on the Barcode of Life Database (BOLD), it was impossible to assign the majority of OTUs to formally described species with binominal nomenclature with our assignment criteria. For our 35 most common OTUs, 7 OTUs 
matched to reference sequences only at family, 14 OTUs to genus, and 14 OTUs to species level (see Additional file 5 for all details). Furthermore, it is notable that the reference sequences with the best matches for OTU 2 (99.52\%), OTU 138 (97.36\%), OTU 466 (98.06\%), and OTU 316 (98.32\%) all bear the same species name Brillia bifida. The same case was observed for OTU 20 and 29 , which best match to reference sequences belonging to Polypedilum convictum with a match of 99.52 and $100 \%$, respectively. Whereas only one OTU bearing the name $B$. bifida was obtained when clustering was based on a $5 \%$ threshold, still, two separate OTUs were found for P. convictum ( $99.52 \%$ and $100 \%$ similarity to reference sequences, respectively). When statistically reanalyzed the one $B$. bifidia, OTU responded the same way as the individual separate OTUs, i.e., being insensitive to manipulation in the streambed and decreasing in the leaf litter with reduced flow velocity (ANOVA, flow velocity, $p<0.01$; partial $\eta^{2}=0.251$ ). In addition, the individual response patterns of both $P$. convictum OTUs were unaffected by the different clustering thresholds.

\section{Discussion}

\section{Chironomidae richness}

Our experiment revealed that the Chironomidae specimens collected from the Felderbach comprised a total of 183 operational taxonomic units (OTUs). It is not straightforward to assign species to OTUs given incomplete reference databases, because using a fixed threshold of $3 \%$ as used in our study is prone to over split genetically diverse species into more than one entity. However, it needs to be emphasized that the high number of OTUs found in this study originates from only one site in one stream and one season. Moreover, even when using a much more conservative OTU clustering threshold of $5 \%$ that has been suggested to be more appropriate for Chironomidae species delimitation [39], still, 142 OTUs are found to co-exist in this stream. According to Cranston [13], a richness of more than 100 chironomid species is not uncommon, especially in lotic environments, unclear if the scale of the lotic environment refers to a stream, a stream and its tributaries, a catchment or just one site of the stream. Concordantly, a high number of Chironomidae species are also documented in the long-term data from the first-order stream Breitenbach in Germany, where over 100 species were recorded for different stream sites [72]. A comparison to the number of only 745 Chironomidae species recorded for Germany [62] suggests either a high number of species that are widely distributed ecological generalists or, more likely, that very many chironomid species are still undescribed.

\section{Comparison of stressor effects on family and OTU level- taxonomic (in)sufficiency?}

On family level, Chironomidae abundance increased with added fine sediment and reduced flow velocity in the streambed but decreased upon flow reduction in the leaf litter (see [5]). In case the concept of taxonomic sufficiency can be applied for this study, the same response patterns should be detected for the majority of OTUs. In sharp contrast, analyzing stressor responses at OTU level revealed not 2 but 15 different stressor response patterns among only the 35 most common (i.e., with the highest overall read abundances) OTUs, ranging from (a) not responding to any experimental manipulation to (b) being affected by one factor to (c) additive effects, and (d) even complex interactions. This clearly shows that the diversity of possible responses to environmental disturbance is not reflected on family level and that the concept of taxonomic sufficiency is insufficient for this study on Chironomidae. However, despite many insensitive OTUs, it is notable that from the sensitive OTUs of the streambed (45.8\% for fine sediment, $37.5 \%$ for flow velocity), almost all responded in the same direction as indicated by the family level response (i.e., increased upon fine sediment addition or flow reduction, respectively). The same pattern was observed for the leaf litter, where $52 \%$ of the OTUs responded negatively to flow reduction, as indicated by a decrease in abundance on family level. In this particular case, analyzing abundances on family level was an indicator that many species or at least high abundant species within the family were affected by the respective stressor. However, as this is a case study, it needs to be validated if this is a general trend or an isolated case. In this regard, OTU 16 represents a counterexample to the overall picture as it decreased and not increased upon fine sediment addition in the streambed. In addition, OTU-level-based analysis revealed that salinity had an effect on single OTUs (one in the streambed and three in the leaf litter) and fine sediment on one OTU in the leaf litter (OTU 30). Furthermore, analyzing stressor effects on single chironomid OTUs showed that stressor effects can be complex and far beyond observable for a pooled family metric. All these single response patterns go unnoticed when the stressor response is analyzed on coarse taxonomic level. Similar results were observed for the mayfly genus Deleatidium in New Zealand, where analyzing single OTUs revealed different stressor response patterns for different OTUs, whereas no effect was observed on genus level [41]. It needs to be highlighted that the 15 different response patterns in this study were observed for the 35 common OTUs manipulating only three factors with two factor levels each. Stream ecosystems, however, can be impacted by a multitude of stressors with a wide range of respective intensities. Even if profound 
data on how single species respond to different stressors would be available, which is clearly not the case for many taxa, multiple stressors can act in surprising ways that cannot always be predicted based on their single stressor effects $[31,50]$. In this regard, an increase in taxonomic resolution means a significant increase in information of how single OTUs respond to environmental changes.

The overall migration from leaf litter to streambed upon water flow reduction as observed on family level [5] could not be confirmed on OTU level. Except for OTU 1, there is no overlap of OTUs decreasing in the leaf litter and increasing in the streambed upon flow reduction. It cannot be ultimately excluded that the analysis was insufficient in detecting a migration of OTUs, but the results strongly suggest that the stressor pattern on family level does not reflect the responses of single OTUs.

The concept of taxonomic sufficiency is currently regarded as a pragmatic trade-off that maximizes the speed and comparability of taxonomic lists for as little money as possible at the cost of losing taxonomic resolution. As an example, identifying chironomids to species rather than family level can more than double the identification time when based on morphology [27] and is associated with a large error. Yet, balancing this tradeoff between speed, cost, and precision becomes obsolete when applying DNA-based identification methods. Hence, with the application of DNA-based methods, the concept of taxonomic sufficiency that came largely as a pragmatic compromise has no real application in many cases anymore if determination can be done with DNA barcoding. The approach allows for maximum taxonomic resolution, comes with no or only marginally higher additional costs [19], and sample processing for metabarcoding at species level is fast.

\section{OTU ecology}

The comparison of OTU sequences with the Barcode of life database (BOLD) suggests that only a very small fraction of OTUs can be assigned reliably to formally described species with binominal nomenclature at this time. Even though this situation will improve in the future, when more reference sequences are released, assigning species names to chironomid OTUs will be an ongoing problem as comparisons with sequence databases might provide contradicting and puzzling results (as observed for Chironomidae, [7]. It needs to be considered if studying autecology is sufficient on OTU level in case a species name cannot be assigned. The potential of studying ecology on OTU level is maybe best being demonstrated not by the case, where no species can be assigned to an OTU, but by the reverse cases of several OTUs being assigned to the same species. In this study, the sequences of four OTUs $(2,138,316$, and 466) match best to reference sequences on BOLD that all bear the name Brillia bifida. For other Chironomidae species, a comparably high species delineation threshold (e.g., for the genus Tanytarsus; [39]) has been suggested and all four OTUs here might very well represent $B$. bifida from a morphological point of view. Thus, one could treat these four OTUs as one response variable in the stressor analysis. While two of the four OTUs (2 and 466) were only analyzed in the streambed and were both insensitive to the experimental manipulation, all four OTUs in the leaf litter responded negatively to flow reduction. In this case, pooling the four OTUs prior to stressor analysis would have delivered the same result, which was confirmed by a second statistical analysis based on the $5 \%$ clustering data. In contrast, OTU 20 and OTU 29, which both correspond to Polypedilum convictum, show a different response to flow reduction in the leaf litter and pooling these according to a different threshold would have changed the biological interpretation. While our study cannot clarify species status of these OTUs, the data can reveal differences between closely related OTUs.

It might be of insignificance if two molecular entities represent (a) distinct species, (b) different 'ecotypes' or 'evolutionary significant units' or (c) separated populations of which members are still capable of reproducing with members of the other population. Diversity and responding to changing environmental conditions are not limited to distinct species, as also single individuals with a different genetic setup (intraspecific variation) can be capable of coping differently with different environmental conditions. Hence, OTU-based analysis ideally even down to unique-sequence-based haplo- or genotypes hold great potential to extend the traditional morphospecies-based view and should be favored. This is because the data are compatible with morphospecies or higher level (genus, family) data but (i) capture also morphologically cryptic species [41] and (ii) also allow detecting changes inside species [20]. Based on OTU, haplo- or genotype data, different fitness values of individual ecotypes, sub-species, or populations can be identified $[36,51]$, which substantially extends our view of biotic responses to environmental factors. While it is possible to use OTUs as entities in ecological studies, it is of indisputably greater strengths if these OTUs can be assigned to formally described species. Only by this, the full available species knowledge can be connected to the OTU-based analyses. Thus, this should be a clear target for future integrative molecular ecological studies.

\section{OTU 1-Rheocricotopus fuscipes (Kieffer, 1909)}

Most reads were assigned to OTU 1, which represents Rheocricotopus fuscipes. $R$. fuscipes is widely distributed in Europe [62] and can occur in densities of more than 
8.000 larvae $/ \mathrm{m}^{2}$ [40]. The high read abundance $(29.6 \%$ of all reads) suggests that $R$. fuscipes was also highly abundant in this study. OTU 1 was well represented in both of the study's microhabitats, streambed, and leaf litter, which is in agreement with the described broad range of microhabitats for $R$. fuscipes (stones, gravels, plants, coarse detritus, sandy bottoms; reviewed in [44]). In contrast to other studies, where R. fuscipes is commonly found in fast-flowing streams [38, 59], an increase of OTU 1 was observed upon flow reduction in the streambed. Despite being common in fast-flowing waters in Central Europe, the species occurs in numerous habitats including the littoral zone of lakes, suggesting a preference for high oxygen levels rather than high flow rates $[38,40]$. The increase in read abundance for this species in the streambed might be explained by immigration of specimens from the leaf litter, where a notable decline was observed. Possibly, reduced flow velocity resulted in a decrease in oxygen in the leaf litter forcing specimens to leave the unfavorable microhabitat.

\section{OTU 2-Brillia bifida (Kieffer, 1909)}

OTU 2 corresponds to Brillia bifida. Larvae of B. bifida are mainly found on decaying leaves [70, 71], which they are suspected to feed on [12]. This is in agreement with the high read abundance of OTU 2 found in the leaf litter microhabitat compared to the streambed. Interestingly enough, $B$. bifida is never found in high densities [44], but contributes the second most reads in this study. This can either be explained by a comparably high biomass of B. bifida and a high primer binding efficiency compared to other chironomid species in our experiment (i.e., they overproportionally contribute to the number of total reads) or poses a contrasting ecological information to the known literature. The species has been noticed to be reasonably independent to current velocity [44], which is only partly in agreement with our results as the abundance decreased in the leaf litter when flow velocity was reduced but was unaffected in the streambed. While OTU 2 did not respond to fine sediment or salinity manipulation in this experiment, B. bifida is a species to be found in no or only little polluted water [45], which highlights the importance of studying the effects of different stressors, as even species sensitive to one stressor can be insensitive to another and vice versa.

\section{OTU 13-Tvetenia calvescens (Edwards, 1929)}

OTU 13 is Tvetenia calvescens with certainty. In our study, this species was almost exclusively found in the leaf litter. This stands in contrast to the literature that describes $T$. calvescens as being abundant on gravel, stones, plants, and mosses (reviewed by [44]). These findings indicate that the streambed might have been already unsuitable as a habitat even without fine sediment addition. T. calvescens is usually the most common species of the genus in streams with flow velocities of $0.5-1.0 \mathrm{~m} / \mathrm{s}$, can be found in stretches with slower currents, and is absent in typical lowland brooks with slower currents (reviewed by [44] T. calvescens is suspected to be sensitive to low oxygen content [44]. The overall low read number assigned to $T$. calvescens in this study indicates that this species was rare. The low abundance might be explained by the general low flow velocity $(16.5 \mathrm{~cm} / \mathrm{s})$ which is supported by even lower read abundances for this species for the reduced flow velocity $(9.6 \mathrm{~cm} / \mathrm{s})$ treatment.

\section{OTU 28-Prodiamesa olivacea (Meigen, 1818)}

OTU 28 corresponds to Prodiamesa olivacea, which can be easily described in all stages [44], resulting in more extensive ecological information being available on this species. Larvae of P. olivacea are bottom inhabitants and are rarely found on stones or among vegetation [71]. They can be numerous in organic silt and over-represented in sand with coarse and fine detritus [70]. The results of this study are in broad agreement with the aforementioned findings, as P. olivacea was almost exclusively found in the streambed and increased further with added fine sediment. In addition, P. olivacea responded positively to reduced flow velocity in this study. Even though the larvae are scarce in stagnant water, they are known to thrive well in stretches with a slow flow when enough decomposing material and silt is available [44]. Despite rarely being found in brackish waters and being classified as haloxenic ("salinity tolerance class 1"; $[44,49,63]$ ), the species was not affected by an increase in salinity in this study, suggesting that it can tolerate a certain increase in salinity.

\section{OTU 3-Micropsectra pallidula (Meigen, 1830)}

OTU 3 corresponds to the M. pallidula, which is difficult or impossible to identify at larval stage [52]. No extensive ecological information is available on this species. However, it is known to inhabit oligotrophic lakes and to be common in silt of rivers in Central Europe [26, 52]. Many Micropsectra larvae are oxygen-demanding, rheophilous species, but some species, i.e., M. atrofasciata (Kieffer, 1911) can tolerate a wide range of substrates, current velocities, and even some shifts in salinity $[26,49]$, which is probably also true for M. pallidula that did not respond to any experimental manipulations in this study. In this case, DNA-based identification made it possible to gather these autecological information, which would have otherwise not been accessible. 


\section{Evidence for indirect effects}

It was beyond the scope of this study to discuss the stressor response of every single OTU. It would also not have been possible, because only few OTUs can be reliably assigned to species and partly because there is a lack of ecological information. However, it is very likely that the applied stressors had an indirect effect on most chironomid OTUs rather than a direct physiological effect. The results of Beermann et al. [5] showed that many Ephemeroptera, Plecoptera, and Trichoptera taxa decrease in abundance upon added fine sediment and reduced flow velocity. Since many chironomids have a higher tolerance for more extreme conditions and the competition decreases with the applied stressors, they are likely increasing in abundance. A further indirect ecological effect might be due to the experimental manipulation affecting the microbial community. Microbes, i.e., bacteria and fungi, are the nutritional basis for many invertebrates either directly as food or indirectly as many invertebrates rely on microbial processes like decomposition of leaf litter. This highlights the need to extend multiple stressor analyses as done here to microbial taxa to understand community responses to multiple stressors and identify the relevance of indirect ecological effects.

\section{Limitations and outlook}

One of the biggest limitations of using DNA metabarcoding for multiple stressor research is that read abundances do not reflect species abundance, but are influenced by species biomass, mitochondrial copy numbers, and primer efficiency. Exact abundances are not being picked up especially for multicellular organisms [15]. Therefore, stressor analyses, as conducted here, do not allow for quantifying changes in species abundance upon respective experimental manipulations or anthropogenic stressors. Hence, additional data on species and life-stage biomass would be beneficial for metabarcoding studies to approximate species abundance. Furthermore, the options of PCR-free methods such as mitochondrial metagenomics [10] might greatly improve biomass and abundance estimations, but need to be studied further as there is scarce information on factors that influence the number of mitochondria per unit of biomass (season, life stage, active versus inactive species). The application of specific strategies to enrich mitochondria in metagenomic studies [42] can be helpful in principle, yet challenges may arise, because specimens have to be processed immediately and cannot be stored prior to laboratory processing. In any case, it needs to be considered if the benefit of taxonomic resolution and being able to analyze single OTUs outweighs the disadvantage of not having exact abundances, which will be greatest for taxa that are small, highly abundant, have a difficult morphology or for which there is a lack of experts such as Chironomidae, Acari, Nematoda and Oligochaeta.

Another limitation of using DNA metabarcoding for analyzing the effects of environmental disturbances to species is that the molecular method does not provide any information on the life stage of the detected species. However, different responses to stressors for the same OTUs might be observed, when different life stages are investigated (e.g., Kefford et al.[35]), that might be visually recognized when identification is based on morphology. Furthermore, different life stages are always encountered when field experiments are conducted in different seasons, but are rarely distinguished. For biomonitoring on the other hand this should be a minor problem, as sampling is conducted at the same time of the year annually in many biomonitoring programs (e.g., the Water Framework Directive, WFD [23], Europe).

Pinder [56] stated for Chironomidae that "specific identification may not [..] be justified for routine monitoring purposes, at least within the limitations imposed by the present state of knowledge regarding the ecological preferences and tolerances of most species." However, chironomids have already been shown to be of value for freshwater biomonitoring $[48,61]$. Data as generated here and elsewhere [2] have the potential to greatly improve studying autecology of species, as it is not hindered by taxonomic impediment. With a more profound understanding of single species ecology, taxa that are widely neglected or only used on a superficial taxonomic level for biomonitoring might be utilized as bioindicators. With the variety of stressor response patterns found here, this study adds further support that Chironomidae specimens should not be treated as one taxon in biomonitoring programs and stressor research.

\section{Conclusion}

With more than 180 OTUs, we revealed a very high chironomid diversity with DNA metabarcoding in a multiple stressor experiment conducted at a single low mountain stream in Germany. The multiple stressor responses at OTU-level, and not only at family level as traditionally done, demonstrate the many and very different response patterns for individual chironomid OTUs (15 response patterns for the 35 most abundant OTUs). Thus, our study highlights the power of increased taxonomic resolution and adds ecological information made accessible by metabarcoding. 


\section{Additional files}

Additional file 1. Chironomid specimen abundance, dry mass, DNA concentrations, and library details.

Additional file 2. Primer combinations.

Additional file 3. Publically available chironomid $\mathrm{COI}$ sequences from specimens sampled in Germany from BOLD(downloaded 01.06.2017).

Additional file 4. Read abundances and standardization of read numbers.

Additional file 5. Taxonomic assignment of the 35 most common chironomid OTUs.

Additional file 6 . Sequencing reads assigned to other taxonomic groups.

\section{Authors' contributions}

Study design: $A B, F L, V E$, and $V Z$; lab work: $A B$ and $V Z$; bioinformatics: $V E$ and $V Z$; data analysis: $A B$ and $F L$; writing the manuscript: $A B, F L, V B$, and VZ. All authors read and approved the final manuscript.

\section{Author details \\ ${ }^{1}$ Aquatic Ecosystem Research, University of Duisburg-Essen, Universitätsstraße 5, 45141 Essen, Germany. ${ }^{2}$ Centre for Water and Environmental Research (ZWU), University of Duisburg-Essen, Universitätsstraße 2, 45141 Essen, Germany. ${ }^{3}$ Present Address: Centre for Biodiversity Genomics, University of Guelph, 50 Stone Road East, Guelph, ON N1G 2W1, Canada. ${ }^{4}$ Department of River Ecology and Conservation, Senckenberg Research Institute and Natu- ral History Museum, 63571 Gelnhausen, Germany.}

\section{Acknowledgements}

We thank Alexander M. Weigand for helpful input on the data analysis, Svenja Karnatz, Li Ma and Julien Utech for sample sorting, Andre Wlecklik, Janis Neumann and Christoph Thiel for assisting with field work, Jens Boenigk and Sabina Marks for helping us with tissue grinding and Jeremy Piggott and Christoph Matthaei for continuous support with ExStream studies. We further thank five anonymous reviewers for their helpful comments and suggestions.

\section{Competing interests}

The authors declare that they have no competing interests.

\section{Availability of data and materials}

All sequences generated in this study are deposited at NCBI (SRA accession SRP154194).

\section{Consent for publication \\ Not applicable.}

\section{Ethics approval and consent to participate}

This study does not involve experimental research on vertebrates or any regulated invertebrates.

\section{Funding}

A.J.B., V.E., and F.L. were supported by the Kurt Eberhard Bode Foundation (GeneStream project). VZ is member of the German Barcode of Life (GBOL) project, which is generously supported by a grant from the German Federal Ministry of Education and Research (FKZ 01L11 101 and 01LI1501). The work is part of EU COST Action CA15219 (DNAqua-Net).

\section{Publisher's Note}

Springer Nature remains neutral with regard to jurisdictional claims in published maps and institutional affiliations.

Received: 25 April 2018 Accepted: 11 July 2018

Published online: 24 July 2018

\section{References}

1. Andersen T, Baranov V, Hagenlund LK, Ivkovic M, Kvifte GM, Pavlek M (2016) Blind flight? A new Troglobiotic Orthoclad (Diptera, Chironomidae) from the Lukina Jama-Trojama Cave in Croatia. PLoS ONE 11(4):e0152884. https://doi.org/10.1371/journal.pone.0152884

2. Andújar C, Arribas P, Gray C, Bruce C, Woodward G, Yu DW, Vogler AP (2018) Metabarcoding of freshwater invertebrates to detect the effects of a pesticide spill. Mol Ecol 27(1):146-166

3. Armitage PD, Cranston PS, Pinder LCV (1995) The Chironomidae: biology and ecology of non-biting midges. The University of Chicago Press, London

4. Ashe P, Murray D (1987) Reiss F The zoogeographical distribution of Chironomidae (Insecta: Diptera). Ann de Limnol Int J Limnol 1:27-60

5. Beermann AJ, Elbrecht V, Karnatz S, Ma L, Matthaei CD, Piggott JJ, Leese F (2018) Multiple-stressor effects on stream macroinvertebrate communities: a mesocosm experiment manipulating salinity, fine sediment and flow velocity. Sci Total Environ 610:961-971

6. Berg MB (1995) Larval food and feeding behaviour. In: Armitage PD, Cranston PS, Pinder LCV (eds) The Chironomidae. Chapman and Hall, London, pp 136-168

7. Brodin Y, Ejdung G, Strandberg J, Lyrholm T (2013) Improving environmental and biodiversity monitoring in the Baltic Sea using DNA barcoding of Chironomidae (Diptera). Mol Ecol Resour 13(6):996-1004. https:// doi.org/10.1111/1755-0998.12053

8. Bucklin A, Steinke D, Blanco-Bercial L (2011) DNA barcoding of marine metazoa. Ann Rev Mar Sci 3:471-508. https://doi.org/10.1146/annurevmarine-120308-080950

9. Carew ME, Pettigrove VJ, Metzeling L, Hoffmann AA (2013) Environmental monitoring using next generation sequencing: rapid identification of macroinvertebrate bioindicator species. Front Zool 10(1):45

10. Choo LQ, Crampton-Platt A, Vogler AP (2017) Shotgun mitogenomics across body size classes in a local assemblage of tropical Diptera: Phylogeny, species diversity and mitochondrial abundance spectrum. Mol Ecol 26:5086-5089

11. Coffman WP (1995) Conclusions. In: Armitage PD, Cranston PS, Pinder LCV (eds) The Chironomidae. Chapman and Hall, London, pp 437-447

12. Cranston P, Oliver D, Sæther O (1989) Keys and diagnoses of the adult males of the subfamily Orthocladiinae (Diptera, Chironomidae). Entomol Scand Suppl 34(165):352

13. Cranston PS (1995) Introduction. In: Armitage PD, Cranston PS, Pinder LCV (eds) The Chironomidae. Chapman and Hall, London, pp 1-7

14. Edgar RC (2013) UPARSE: highly accurate OTU sequences from microbial amplicon reads. Nat Methods 10(10):996-998

15. Elbrecht $V$, Leese F (2015) Can DNA-based ecosystem assessments quantify species abundance? Testing primer bias and biomass-sequence relationships with an innovative metabarcoding protocol. PLOS ONE 10(7):e0130324

16. Elbrecht V, Leese F (2017) PrimerMiner: an R package for development and in silico validation of DNA metabarcoding primers. Methods Ecol Evol 8(5):622-626

17. Elbrecht V, Leese F (2017) Validation and development of COI metabarcoding primers for freshwater macroinvertebrate bioassessment. Front Environ Sci 5:11

18. Elbrecht V, Peinert B, Leese F (2017) Sorting things out-assessing effects of unequal specimen biomass on DNA metabarcoding. PeerJ Preprints,

19. Elbrecht V, Vamos EE, Meissner K, Aroviita J, Leese F (2017) Assessing strengths and weaknesses of DNA metabarcoding-based macroinvertebrate identification for routine stream monitoring. Methods Ecol Evol 8:1265-1275

20. Elbrecht V, Vamos EE, Steinke D, Leese F (2018) Estimating intraspecific genetic diversity from community DNA metabarcoding data. PeerJ $6: e 4644$

21. Ellis D (1985) Taxonomic sufficiency in pollution assessment. Mar Pollut Bull 16(12):459

22. Emilson CE, Thompson DG, Venier LA, Porter TM, Swystun T, Chartrand D, Capell S, Hajibabaei M (2017) DNA metabarcoding and morphological macroinvertebrate metrics reveal the same changes in boreal watersheds across an environmental gradient. Sci Rep 7:12777. https://doi. org/10.1038/s41598-017-13157-x 
23. European Union (2000) Directive 2000/60/EC of the European Parliament and of the Council of 23 October 2000 establishing a framework for Community action in the field of water policy. Off J Eur Union L327:1-73

24. Ferrington LC, Blackwood MA, Wright CA, Crisp NH, Kavanaugh JL, Schmidt FJ (1991) A protocol for using surface-floating pupal exuviae of Chironomidae for rapid bio assessment of changing water quality. In: Sediment and stream water quality in a changing environment: trends and explanation. Proceedings of the Vienna symposium, August 1991. IAHS Publ. no. 203, pp 181-190

25. Gibson JF, Shokralla S, Curry C, Baird DJ, Monk WA, King I, Hajibabaei M (2015) Large-scale biomonitoring of remote and threatened ecosystems via high-throughput sequencing. PLoS ONE 10(10):e0138432. https://doi. org/10.1371/journal.pone.0138432

26. Giłka W (2011) Ochotkowate-Chironomidae, plemię: Tanytarsini, postaci dorosłe, samce. Klucze do oznaczania owadów Polski. [Non-biting midges—Chironomidae, tribe Tanytarsini, adult males. Keys for the Identification of Polish Insects]. Nr 177 serii kluczy. Część XXVIII, MuchówkiDiptera, zeszyt 14b. Polskie Towarzystwo Entomologiczne. Biologica Silesiae, $95 \mathrm{pp}$

27. Hawkins C, Norris R Effects of taxonomic resolution and use of subsets of the fauna on the performance of RIVPACS-type models. Assessing the biological quality of fresh waters: RIVPACS and other techniques. In: Proceedings of an international workshop held in Oxford, UK, on 16-18 September 1997, 2000. Freshwater Biological Association (FBA), pp 217-228

28. Hebert PD, Stoeckle MY, Zemlak TS, Francis CM (2004) Identification of birds through DNA Barcodes. PLoS Biol 2(10):e312. https://doi. org/10.1371/journal.pbio.0020312

29. Hebert PDN, Cywinska A, Ball SL, DeWaard JR (2003) Biological identifications through DNA Barcodes. Proc R Soc B Biol Sci 270(1512):313-321. https://doi.org/10.1098/rspb.2002.2218

30. Hilsenhoff WL (1966) The biology of Chironomus plumosus (Diptera: (hironomidae) in Lake Winnebago, Wisconsin. Ann Entomol Soc Am 59(3):465-473

31. Jackson JK, Fureder L (2006) Long-term studies of freshwater macroinvertebrates: a review of the frequency, duration and ecological significance. Freshwater Biol 51(3):591-603. https://doi.org/10.111 $1 / j .1365-2427.2006 .01503 . x$

32. Johnson RK, Boström B, van de Bund W (1989) Interactions between Chironomus plumosus (L.) and the microbial community in surficial sediments of a shallow, eutrophic lake. Limnol Oceanogr 34(6):992-1003

33. Jones FC (2008) Taxonomic sufficiency: the influence of taxonomic resolution on freshwater bioassessments using benthic macroinvertebrates. Environ Rev 16:45-69

34. Kallimanis AS, Mazaris AD, Tsakanikas D, Dimopoulos P, Pantis JD, Sgardelis SP (2012) Efficient biodiversity monitoring: which taxonomic level to study? Ecol Indic 15(1):100-104

35. Kefford BJ, Nugegoda D, Zalizniak L, Fields EJ, Hassell KL (2007) The salinity tolerance of freshwater macroinvertebrate eggs and hatchlings in comparison to their older life-stages: a diversity of responses. Aquat Ecol 41(2):335-348

36. Ki Kojima (1971) Is there a constant fitness value for a given genotype? NO! Evolution 25(2):281-285

37. Landeiro VL, Bini LM, Costa FR, Franklin E, Nogueira A, de Souza JL, Moraes J, Magnusson WE (2012) How far can we go in simplifying biomonitoring assessments? An integrated analysis of taxonomic surrogacy, taxonomic sufficiency and numerical resolution in a megadiverse region. Ecol Indic 23:366-373

38. Lehman J (1971) Die Chironomiden der Fulda. Sytematische, ökologische und faunistische Untersuchngen. Arch Hydrobiol Suppl 37:466-555

39. Lin $X$, Stur E, Ekrem T (2015) Exploring genetic divergence in a speciesrich insect genus using 2790 DNA Barcodes. PLoS ONE 10(9):e0138993

40. Lindegaard C, Thorup J, Bahn M (1975) The invertebrate fauna of the moss carpet in the Danish spring Ravnkilde and its seasonal, vertical, and horizontal distribution. Arch Hydrobiol 75:109-139

41. Macher JN, Salis RK, Blakemore KS, Tollrian R, Matthaei CD, Leese F (2016) Multiple-stressor effects on stream invertebrates: DNA barcoding reveals contrasting responses of cryptic mayfly species. Ecol Indic 61:159-169. https://doi.org/10.1016/j.ecolind.2015.08.024
42. Macher JN, Zizka VMA, Weigand AM, Leese F (2018) A simple centrifugation protocol for metagenomic studies increases mitochondrial DNA yield by two orders of magnitude. Methods Ecol Evol 9(4):1070-1074

43. Martin M (2011) Cutadapt removes adapter sequences from highthroughput sequencing reads. EMBnet J 17(1):10-12

44. Moller Pillot H (2014) Chironomidae Larvae: Orthocladiinae, 3rd edn. Brill, Leiden

45. Moog O (1995) Fauna aquatica austriaca. Wasserwirtschaftskataster, Bundesministerium für Land-und Forstwirtschaft, Wien

46. Mueller M, Pander J, Geist J (2013) Taxonomic sufficiency in freshwater ecosystems: effects of taxonomic resolution, functional traits, and data transformation. Freshw Sci 32(3):762-778

47. Nakagawa S, Cuthill IC (2007) Effect size, confidence interval and statistical significance: a practical guide for biologists. Biol Rev 82(4):591-605. https://doi.org/10.1111/j.1469-185X.2007.00027.x

48. Orendt C, Garcia X-F, Janecek B, Michiels S, Otto C-J, Müller R (2014) Faunistic overview of Chironomidae (Insecta: Diptera) in lowland running waters of north-east Germany (Brandenburg) based on 10-year EU-Water Framework Directive monitoring programme. Lauterbornia 77:37-62

49. Orendt C, Spies M (2012) Chironomini (Diptera: Chironomidae) Keys to Central European larvae using mainly macroscopic characters, 66th edn. NHBS, Brandvorwerkstr, p 04275

50. Ormerod SJ, Dobson M, Hildrew AG, Townsend CR (2010) Multiple stressors in freshwater ecosystems. Freshw Biol 55:1-4. https://doi.org/10.111 1/j.1365-2427.2009.02395.x

51. Osier TL, Lindroth RL (2001) Effects of genotype, nutrient availability, and defoliation on aspen phytochemistry and insect performance. J Chem Ecol 27(7):1289-1313

52. Pankratova VY (1983) Larvae and pupae of midges of the subfamily Chironominae of the fauna of the USSR (Diptera, Chironomidae $=$ Tendipedidae). Larvae and pupae of midges of the subfamily Chironominae of the fauna of the USSR (Diptera, Chironomidae $=$ Tendipedidae)

53. Pfenninger M, Nowak C (2008) Reproductive isolation and ecological niche partition among larvae of the morphologically cryptic sister species Chironomus riparius and C. piger. PLoS ONE 3(5):e2157

54. Piggott JJ, Salis RK, Lear G, Townsend CR, Matthaei CD (2015) Climate warming and agricultural stressors interact to determine stream periphyton community composition. Glob Change Biol 21(1):206-222

55. Piggott JJ, Townsend CR, Matthaei CD (2015) Reconceptualizing synergism and antagonism among multiple stressors. Ecol Evol 5(7):1538-1547

56. Pinder LCV (1986) Biology of fresh-water Chironomidae. Annu Rev Entomol 31:1-23

57. Piñol J, Mir G, Gomez-Polo P, Agustí N (2015) Universal and blocking primer mismatches limit the use of high-throughput DNA sequencing for the quantitative metabarcoding of arthropods. Mol Ecol Resour 15(4):819-830

58. Power ME (1990) Effects of fish in river food webs. Science 250(4982):811814. https://doi.org/10.1126/science.250.4982.811

59. Ringe F (1974) Chironomiden-Emergenz 1970 in Breitenbach und Rohrwiesenbach. Schlitzer produktionsbiologische Studien (10). Archiv für Hydrobiologie/Supplement 45(2/3):212-304

60. Rognes T, Flouri T, Nichols B, Quince C, Mahé F (2016) VSEARCH: a versatile open source tool for metagenomics. PeerJ 4:e2584

61. Rosenberg DM (1992) Freshwater biomonitoring and Chironomidae. Netherland Journal of Aquatic Ecology 26(2-4):101-122

62. Sæther O, Spies M (2004) Fauna Europaea: Chironomidae. Fauna Europaea version 1:1

63. Steenbergen H (1993) Macrofauna-atlas van Noord-Holland: verspreidingskaarten en responsies op milieufactoren van ongewervelde waterdieren. Provincie Noord-Holland, Dienst Ruimte en Groen

64. Stein ED, White BP, Mazor RD, Jackson JK, Battle JM, Miller PE, Pilgrim EM, Sweeney BW (2014) Does DNA barcoding improve performance of traditional stream bioassessment metrics? Freshw Sci 33(1):302-311. https:// doi.org/10.1086/674782

65. Sunnucks P, Hales DF (1996) Numerous transposed sequences of mitochondrial cytochrome oxidase $\mathrm{I}-\mathrm{II}$ in aphids of the genus Sitobion (Hemiptera: Aphididae). Mol Biol Evol 13(3):510-524

66. Taberlet P, Coissac E, Pompanon F, Brochmann C, Willerslev E (2012) Towards next-generation biodiversity assessment using DNA metabarcoding. Mol Ecol 21(8):2045-2050. https://doi.org/10.1111/j.1365294X.2012.05470.x 
67. Team RC (2013) R: a language and environment for statistical computing Version 3.1. 3. R Foundation for Statistical Computing, Vienna

68. Terlizzi A, Bevilacqua S, Fraschetti S, Boero F (2003) Taxonomic sufficiency and the increasing insufficiency of taxonomic expertise. Mar Pollut Bull 46(5):556-561. https://doi.org/10.1016/S0025-326X(03)00066-3

69. Theissinger K, Kästel A, Elbrecht V, Leese F, Brühl C (2018) Using DNA metabarcoding for assessing chironomid diversity and community change in mosquito controlled temporary wetlands. Metabarcoding Metagenomics 2:e21060

70. Tolkamp HH (1980) Organism-substrate relationships in lowland streams. Wageningen

71. Verdonschot P, Lengkeek W (2006) Habitat preferences of selected indicators. Alterra, Australia

72. Wagner R, Marxsen J, Zwick P, Cox EJ (2011) Central European Stream ecosystems: the long term study of the Breitenbach. Wiley, Hoboken
73. Ward RD, ZemlakTS, Innes BH, Last PR, Hebert PD (2005) DNA barcoding Australia's fish species. Philos Trans R Soc Lond B Biol Sci 360(1462):18471857. https://doi.org/10.1098/rstb.2005.1716

74. Weiss M, Leese F (2016) Widely distributed and regionally isolated! Drivers of genetic structure in Gammarus fossarum in a human-impacted landscape. BMC Evol Biol 16:153. https://doi.org/10.1186/s12862-016-0723-z

75. Wirta HK, Vesterinen EJ, Hambäck PA, Weingartner E, Rasmussen C, Reneerkens J, Schmidt NM, Gilg O, Roslin T (2015) Exposing the structure of an Arctic food web. Ecol Evol 5(17):3842-3856

76. Zhou X, Kjer KM, Morse JC (2007) Associating larvae and adults of Chinese Hydropsychidae caddisflies (Insecta: Trichoptera) using DNA sequences. J N Am Benthol Soc 26(4):719-742. https://doi.org/10.1899/06-089.1

\section{Submit your manuscript to a SpringerOpen ${ }^{\circ}$ journal and benefit from:}

- Convenient online submission

- Rigorous peer review

- Open access: articles freely available online

- High visibility within the field

- Retaining the copyright to your article

Submit your next manuscript at springeropen.com 